\title{
MATHEMATICAL NOTES
}

PUBLISHED BY

\section{THE EDINBURGH MATHEMATICAL SOCIETY \\ Edited By A: C. AITKEN, M.A., D.Sc., F.R.S.E.}

\section{An Apparatus for Determining Coefficients in Power Series}

\author{
By the late R. W. M. Thomson, B.Sc. (Communicated by \\ Professor Peddie.)*
}

The diagrammatic figure illustrates the principle involved. The vertical full lines represent ordinates of a curve of which the

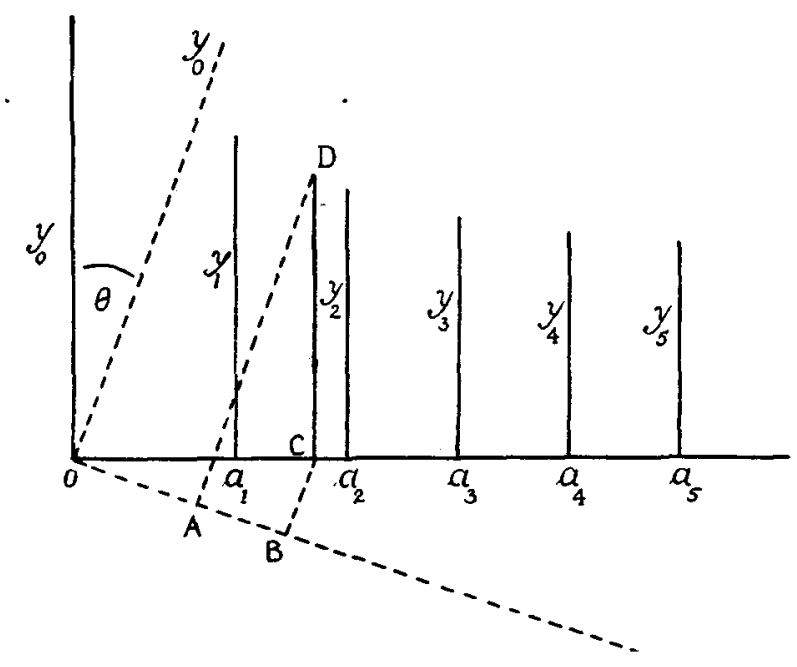

abscissae are $O a_{1}, O a_{2}$, etc. Let now all these vertical lines be rotated round the points $O, a_{1}, a_{2}$, etc., while retaining their original lengths,

* The early death of Mr R. W. M. Thomson, a graduate of University College, Dundee, and a son of the late Mr James Thomson, LL.B., was felt, by those who knew him, to be a loss to science. Soon after his graduation he was appointed to the staff of King's College, London, in the department of Physical Chemistry ; and his early promise as a student was fully maintained in his new post.

He had special aptitude in devising apparatus for experimental work, and found use for it in the course of a chemical investigation in which many experimental curves were obtained. To save the labour of detailed calculation in each case, he devised and constructed the apparatus here described.-W. P. 
through the same angle $\theta$. The curve passing through the new positions of the end points is now to be referred to the tilted axis $O y_{0}$ and the line $O A B$, drawn at right angles thereto. If we put $O C=x, C D=y$, and let $x^{\prime}, y^{\prime}$, be the corresponding coordinates with reference to the tilted axis we get

$$
\begin{aligned}
& y^{\prime}=y+x \sin \theta \\
& x^{\prime}=x \cos \theta
\end{aligned}
$$

Hence, if the original curve, were approximately representable by the equation

$$
y=y_{0}+a_{1} x+a_{2} x^{2}+a_{3} x^{3}+\ldots,
$$

the new curve would represent the relation

$$
y^{\prime}=y_{0}+\left(a_{1}+\sin \theta\right) \sec \theta \cdot x^{\prime}+a_{2} \sec ^{2} \theta \cdot x^{\prime 2}+a_{3} \sec ^{3} \theta \cdot x^{\prime 3}+\ldots
$$

If we write this as

$$
y^{\prime}=y_{0}+a_{1}^{\prime} x+a_{2}^{\prime} x^{\prime 2}+a_{3}^{\prime} x^{\prime 3}+\ldots \text {, }
$$

we obtain, for the new constants, the simple relations

$$
\begin{aligned}
& y_{0}^{\prime}=y_{0}, \\
& a_{1}^{\prime}=\left(a_{1}+\sin \theta\right) \sec \theta, \\
& a_{2}^{\prime}=a_{2} \sec ^{2} \theta, \\
& a_{3}^{\prime}=a_{3} \sec ^{3} \theta, \\
& \ldots \ldots \ldots \ldots \ldots \\
& \cdots \ldots \ldots \ldots \\
& a_{n}^{\prime}=a_{n} \sec ^{\prime} \theta .
\end{aligned}
$$

The photographs show the actual apparatus, as used, in the normal and tilted positions. The constants $y_{0}, a_{1}, a_{2}$, etc., are got from data of measurement once for all. The lower ends of the tiltable rods are pivoted on a subsidary beam which is parallel to the main beam, and the tilt of the rods is produced by sliding the subsidiary beam parallel to itself. The original curve (no tilt) was selected as being suitable for the special set of experimental curves which were dealt with. If the lengths of the rods were made adjustable, other original curves could be used.

Two fixed pins, at a slight distance apart, pass through the upper end of each rod; and two light, flat, steel springs, one at each side of the set of rods, pass through the space between each pair of pins. These springs are clamped together by a pair of clamps which can be seen in the illustrations. The two springs thus act as a single spring, which can slide in the spaces between each pair of pins, and is fixed by clamping screws at the end nearest the 
main beam. This point of fixture can be taken as an additional point on the curve corresponding to $y=0$ on the untilted curve. Half a dozen $V$-shaped notch pieces, two fixed on the longest rod; and four (visible as such in the illustrations) fixed on the main beam or its
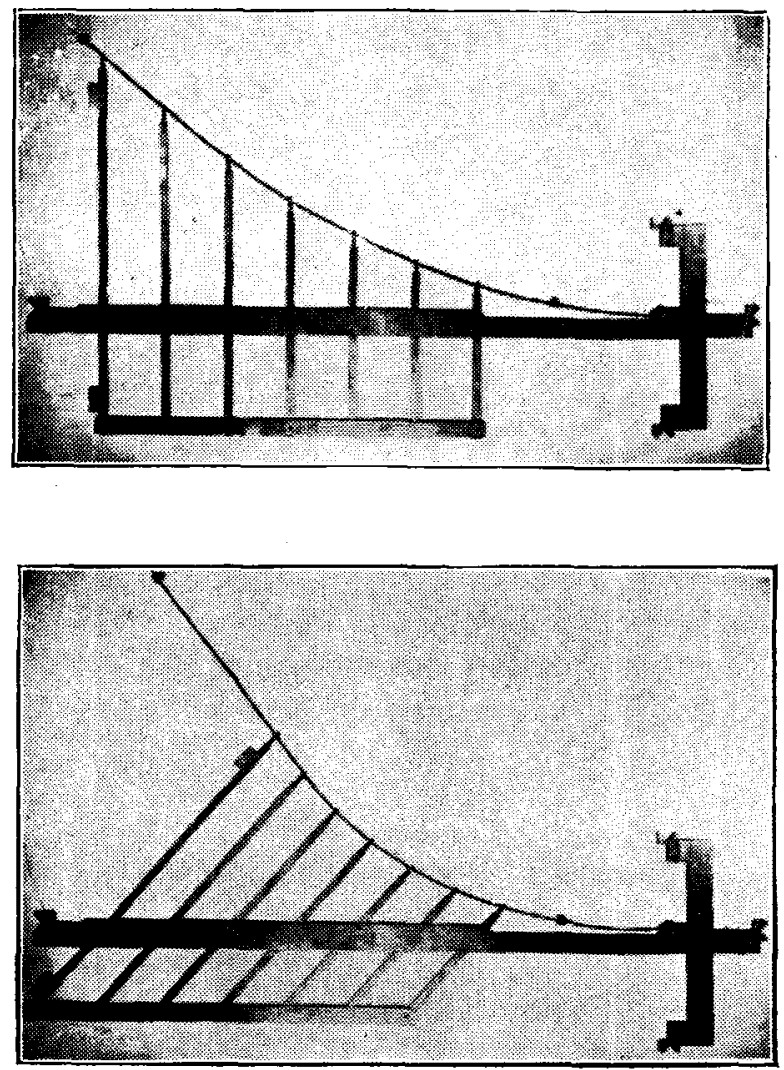

cross piece, serve for adjustment of the apparatus in a vertical framework if desired. Otherwise, the apparatus can lie flat on the paper on which the experimental curve is traced, and adjustment be made until the curve of the spring coincides as closely as may be desired with the experimental curve, to which an empirical formula is thus readily found.

The spring of course merely gives a smooth curve passing through the points fixed by the rods. The diagrams show how very different may be the shape of the curve relatively to the untilted and the tilted axes. The origin for $y^{\prime}$ may clearly be shifted to any desired position along the $y^{\prime}$ axis. 